\title{
Design of ZigBee Data Acquisition System Based on CC2530
}

\author{
Zhaowei Liu ${ }^{1}$, Hongbin $\mathrm{Yu}^{2}$ \\ ${ }^{1,2}$ School of Mechanical Engineering ,Tianjin Polytechnic University, China,300387,
}

\begin{abstract}
In order to avoid the inconvenience caused by the complex environment of industrial production to the data transmission, a data acquisition method is designed, which is more efficientand low power consumption compared to the traditional wireless transmission.Combined with the characteristics of ZigBee technology applied to data acquisition system, the hardware design method of data acquisition node based on CC2530 chip and the network of each node in the network are discussed,including the software design method of adding network.In this paper, we are going to realize the workshop network management, and the computer terminal can detect the status information of multiple devices in real time, the system can collect the state of the equipment in real time based on the CC2530 data acquisition and production information,. The collecting device and the receiving device transmit the status of equipment based on ZigBee wireless communication mode and ZigBee module communicate with the computer terminal through RS-485 null modem cable.
\end{abstract}

Key words: wireless sensor network; ZigBee; data acquisition

\section{Introduction}

In order to improve the production capacity of the whole system, the effective information acquisition technology must be used to gain fully, real-time, accurately obtain information in the process of manufacturing, managing and controlling the production process by taking this as foundation[1].In the actual production, in order to ensure the normal operation of the workshop, the operation status of each equipment should be obtained in real time. In the traditional collection mode, the field data acquisition device connect with each sensor through the RS-485 bus. The poor production environment in the industrial production is very disadvantageous to the wired transmission [2]. Although remote transmission of data can be get through GPRS and Internet to the computer terminal, high power consumption, the difficulties of wiring and equipment installation when the monitoring range is bigger must be taking fully into account.ZigBee wireless transmission of low power consumption, low cost, large network capacity is very consistent with the requirements of the production conditions, so the paper gives the design of ZigBee wireless data acquisition system based on CC2530 RF chip.

\section{Overall Structure Analysis Of Wireless Sensor Networks}

The purpose of this acquisition system is to realize the real-time acquisition, transmission, analysis, feedback and storage of the temperature and vibration frequency of multiple devices[3].Sensors on each machine collect data under the control of the CC2530 module.And the data collected by the sensor will be transmit to the coordinator by wireless RF module. After the coordinator for data processing, the data processed will be transmitted to the PC though the RS-485 serial port and the PC machine display the status parameters in real time. The system is composed of the upper computer and the lower monitoring network, which is connected with the monitoring network through the ZigBee Ethernet protocol converter to realize the data transmission [4]. The monitoring network is composed of the coordinator, the router and the terminal node. PC and the coordinator is placed in the main control room, which is responsible for receiving and processing the data transmitted from the routers and the terminal nodes. PC program receive data for processing and display the data curve and PC will alarm when the abnormal condition monitoring equipment occurs ;Routers and terminal nodes distribute on each device, the terminal node is responsible for data collection and the router is responsible for routing and data transmission. The system of wireless sensor network structure is shown in Figure 1.

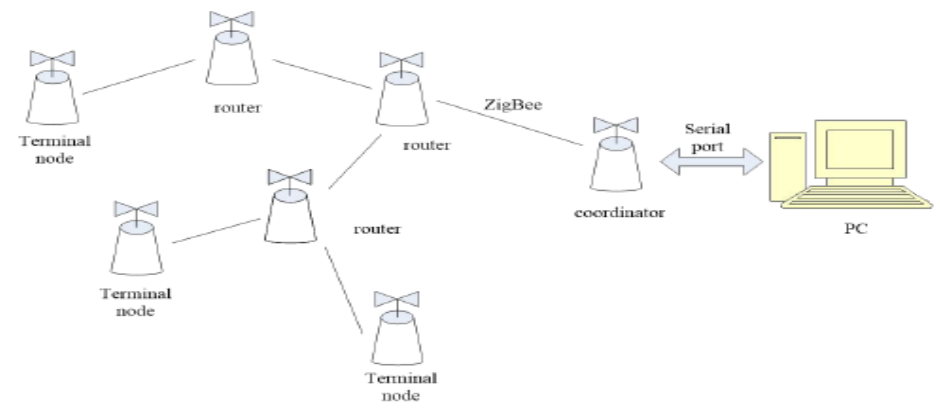

Fig.1 Overall structure analysis of Wireless Sensor Networks 


\section{Node Hardware Design}

In wireless sensor networks, sensor nodes are equipped with various sensors, used to the temperature acquisition device, vibration frequency data and through the network will transmit data to the routing node or coordinator node. Wireless sensor node is the basic composition unit of the wireless network, which is mainly composed of four parts: sensor module, CC2530 control module, CC2530 RF module, power supply module[5].Depending on the actual function, the different nodes will be configured with other functional units, such as the terminal node to add the sensor module, the coordinator node to add the function of the serial port module and other.ZigBee nodes as shown in Figure 2.

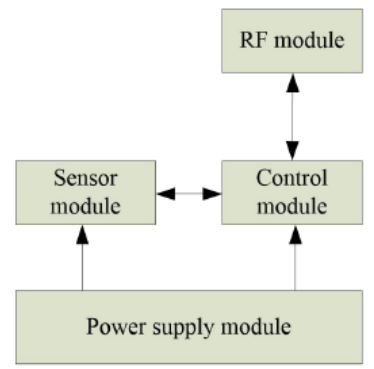

Fig.2 node function structure

\subsection{CC2530 module}

CC2530 is for IEEE802.15.4, system on chip and ZigBee RF4CE application solutions, with excellent performance of RF transceiver and its programming output power as high as $4.5 \mathrm{dBm}$, have high receiver sensitivity and anti-interference ability[6], simple peripheral circuit and small package can be used for the production of smaller nodes; $128 \mathrm{~KB}$ on-chip flash memory to accommodate the ZigBee protocol stack; when the node is idle, that is to say, sleep mode, so as to reduce the energy loss. CC2530 peripheral module mainly includes circuit and filter circuit, crystal oscillator circuit, and RF front-end balun circuit, crystal oscillator circuit is composed of a $32 \mathrm{mhz}$ and $32.768 \mathrm{KHz}$ crystal oscillator circuit, in order to ensure the accuracy of the serial baud rate.

\subsection{Sensor module}

The sensor module is composed of a temperature sensor and a vibration sensor, vibration sensor using module mpu-6050, is the world's first integration of six axis motion processing components, compared to the multi component solutions, eliminating the axis of gyro and accelerator difference, reduce the amount of packaging space. Vibration sensor by the axis of the accelerometer to obtain vibration frequency, using IIC interface, using ordinary microcontroller IO simulation, get the acceleration and the angular velocity of the data. The original data, you can with serial transfer to the host computer display, using the built-in DMP, can get the final data.

\subsection{Power supply module}

In the design of the system, ZigBee node downloading and debugging equipment work with a $5 \mathrm{~V}$ supply and $\mathrm{CC} 2530$ working voltage for the $2 \sim 3.6 \mathrm{~V}$, therefore using the voltage conversion chip to do level conversion. In order to meet the ZigBee nodes may supply demand under different working conditions, the system for ZigBee node provides three types of power supply: battery powered, USB power supply, stable power supply DC power supply.

\section{Software Design}

Wireless sensor system software programming must rely on the hardware platform, the system software design mainly includes the host computer software design and ZigBee node software design two parts.

\subsection{PC software design}

PC control, the software design is in Visual Studio 2010 compiler environment to generate and visual studio 2010 is an integrated development environment visual studio 2010 net framework 2.0 library contains the serialport class, can be convenient to serial communication functions[7]. PC is the core of the monitoring system, for receiving and protocol converter to send data analysis and the data were corresponding processing, machine environment reference for the operating personnel, and data on the detection of abnormal when the alarm. PC program flow chart as shown in Figure 3. 


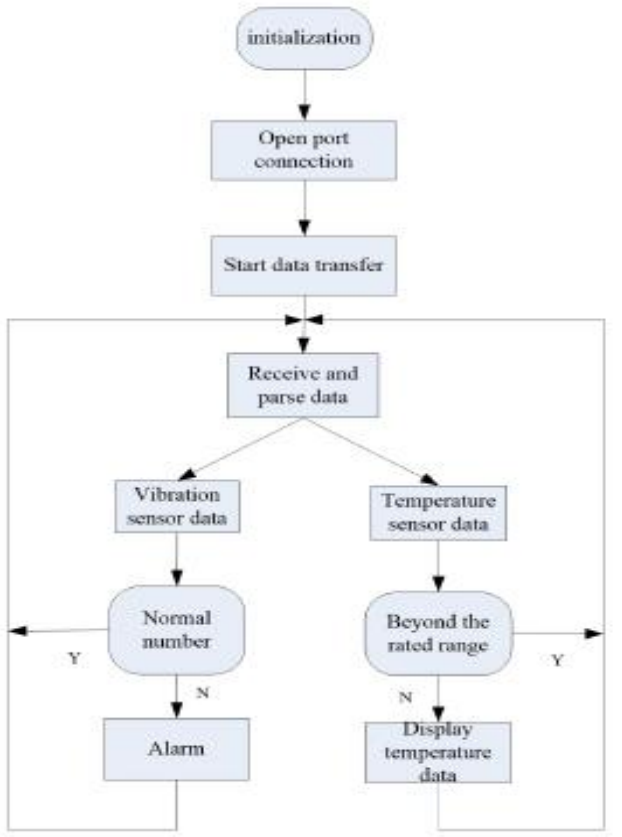

Fig. 3 PC program flow chart

\subsection{ZigBee node software design}

ZigBee node software design mainly includes the terminal node software workflow design, workflow design software routing node and coordinator of workflow software design. Among them, the routing node is responsible for forwarding data collection and data, its working process is shown in Figure 4. The software flow difference between routing node and the terminal node is small; it is mainly determined by the routing function. Compared to the terminal node,the routing node increase the data forwarding in the routing node software flow chart which reflects the received commands to the terminal nodes and the collection of data on the received forwarded to the coordinator.At the same time, the routing node also need to maintain routing list and in order to ensure the correct forwarding of data.
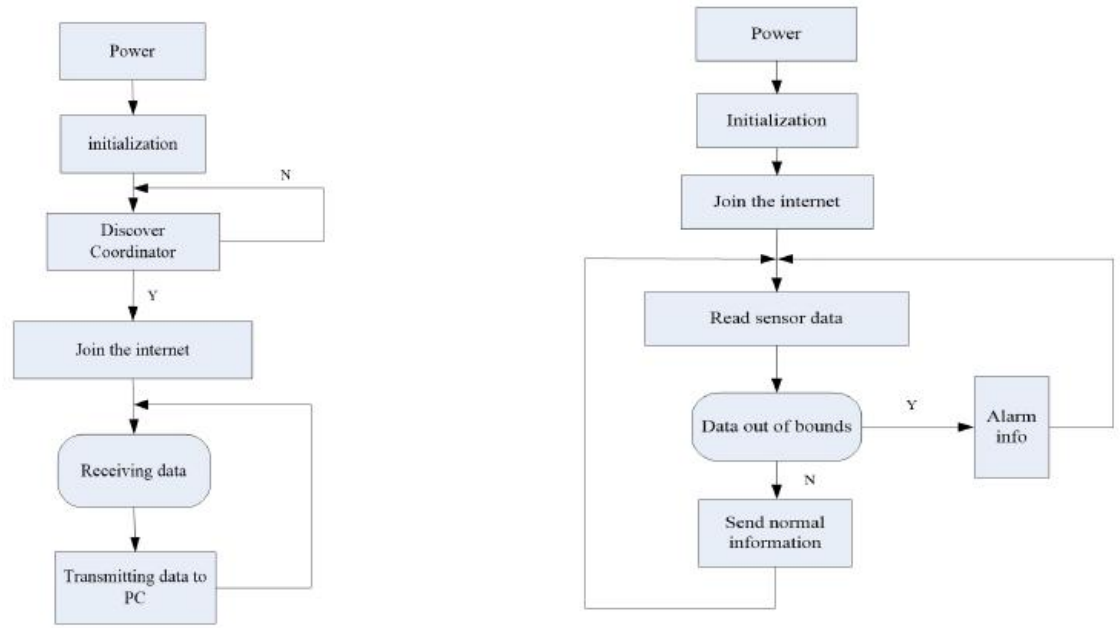

Fig. 4 Routing node workflow Fig.5 Terminal node workflow

The terminal node receives the coordinator instruction and carries on the data collection and the data, its work flow is shown in Figure 5.

First of all, the electric terminal node initialization, join the existing network; if a network failure, enter a dormant state; if join the network, advanced for routing maintenance response, and then to receive the parent instruction; if no instruction node enter dormancy. When the node receives the instruction, analyze the instruction sequence selection with the opening or closing of the sensor sensor, open the corresponding data collection, in order to return the data analysis after the parent node, the parent node is processed in the next step, the node into the next round of route maintenance in response to the instruction data receiving and instruction analysis. In order to enhance the reliability of data acquisition, operation time of frequent sleep state process is processed, which is in the instruction repeatedly in the analysis process, do not open any sensor, just enter a 
dormant state and enter the network [8]. has been dormant. State of the terminal nodes will be periodically wake up, wake up to carry out the route maintenance response and whether the need to receive instruction data checks.

The coordinator node not only for data collection and transmission, and is also responsible for the PC to communicate, its working process is shown in Figure 6. The coordination on the initialization node, then establish a network to establish a network, the coordinator node start processing the join request. Processed nodes join request, the coordinator check serial time there is no need to receive the first data. The first time when there is no need to receive data, and will enter the node join processing program, followed by the cycle. When there is the first data to be received, will receive instruction serial data. The coordinator receives the instruction, through the wireless network to send to other nodes, and then enter the wait for the data link receiving collected, when no data is returned when the coordinator nodes join the program and check by serial port command input when data is returned, coordination The processing device receives the data through the serial port to upload to the host computer and node joined the program. When the last instruction run after, coordination of sensor nodes will in each instruction are running after a serial data receiving inspection, to receive new instructions. When no new instruction needs to receive node is still running a instruction.

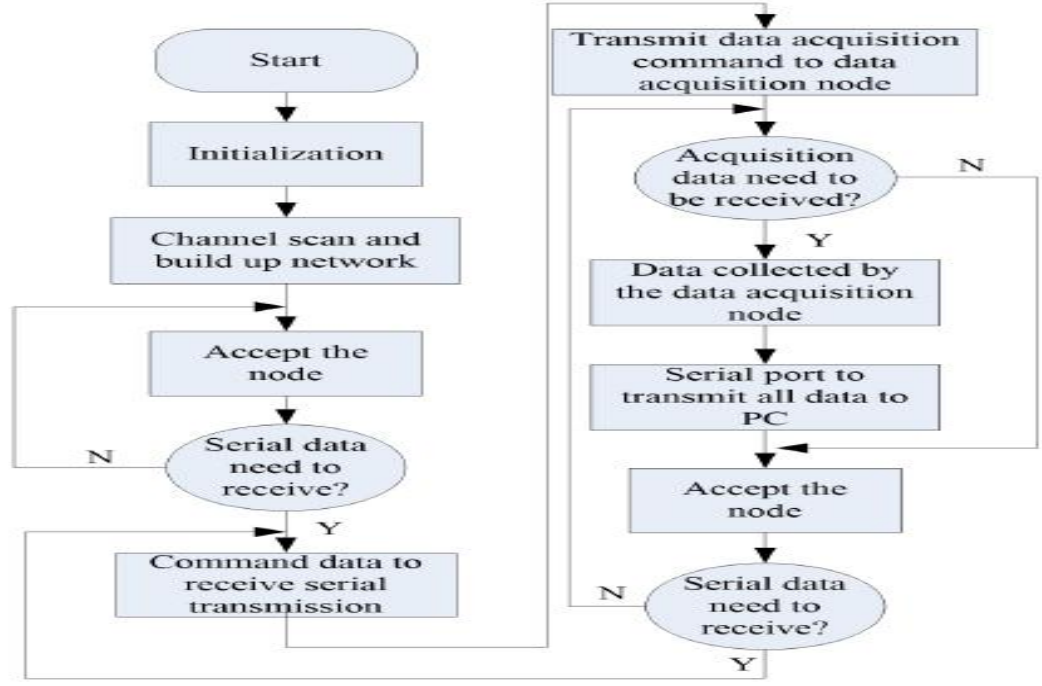

Fig.6 Coordinator node workflow

\section{Summary And Outlook}

Experiment in the two nodes of the most simple way, which a node for data acquisition terminal nodes, another node to the coordinator node. Using the two node network and data acquisition, mainly for machine tool temperature and vibration frequency of the data acquisition, network node, and successfully collected the data, compared with the traditional acquisition methods, signal transmission distance is farther and has higher precision.

\section{References}

[1]. IAN F A ,MEHMET C V. Wireless sensor networks[M]. Hoboken,New Jersey,USA: John Wiley\&Sons, 2010.

[2]. WALTENEGUS D, CHRISTIAN P. Fundamentals of wireless sensor networks: theory and pratice [M]. Hoboken, New Jersey, USA: John Wiley\&Sons, 2010.

[3]. ALThAUS E, CALINESCU G, MANDOIU I, et al.Power efficient range assignment in Ad Hoc wireless networks [C]/ /Proceedings of the 2003 IEEE Conference on Wireless Communication and Networking.Piscataway: IEEE, 2003:1889-1894.

[4]. Cha Yong Dae, Yoon Gilwon. Ubiquitous health monitoring system for multiple users using a ZigBee and WLAN dual-network.[J]. Telemedicine and e-Health, 2009, 159:165.

[5]. Li Junbin, Hu Yongzhong. Design and application of ZigBee communication network based on

[6]. [J]. CC2530 electronic design engineering, 2011, 16:108-111.

[7]. Chen Xuefeng. Algorithm of pulse wave feature extraction and its application [D]. Dalian

[8]. University of Technology, 2006

[9]. Li Xuebo. Research and implementation of the human physiological index detection algorithm based on the optical capacitance integrated pulse wave [D]. Northeastern University, 2011

[10]. Chau-Chung Song,Chen-Fu Feng. Simulation and Experimental Analysis of a ZigBee Sensor Network with Fault Detection and Reconfiguration Mechanism.Proceeding of 2011 8thAsian Control Conference. 2011,5. pp.659-664 